MATEC Web of Conferences 19, 01029 (2014)

DOI: $10.1051 /$ matecconf/ 20141901029

(C) Owned by the authors, published by EDP Sciences, 2014

\title{
Hard- and software of real time simulation tools of Electric Power System for adequate modeling power semiconductors in voltage source convertor based HVDC and FACTS
}

\author{
Ruslan A. Ufa ${ }^{a}$, Venera A. Sulaymanova, and Alexandr S. Gusev \\ National Research Tomsk Polytechnic University, 634050 Tomsk, Russia
}

\begin{abstract}
The motivation of the presented research is based on the needs for development of new methods and tools for adequate simulation of Flexible Alternating Current Transmission System (FACTS) devices and High Voltage Direct Current Transmission (HVDC) system as part of real electric power systems (EPS). For that, a hybrid approach for advanced simulation of the FACTS and HVDC based on Voltage Source is proposed. The presented simulation results of the developed hybrid model of VSC confirm the achievement of the desired properties of the model and the effectiveness of the proposed solutions.
\end{abstract}

\section{Introduction}

The development of power electronics available some answers of currently controlling issues from conversion and transmission of high voltage. There are lots of devices (FACTS - Flexible Alternating Current Transmission System, HVDC - High Voltage Direct Current transmission technologies) newly designed on or reintroduced on the basis of Voltage Source Converter (VSC), caused by the evolution of switches, semiconductor fully controllable for high-speed. However, the widespread installation of such devices is limited and delayed, due to the relatively operating experience and low level of knowledge about their influence on the network. The most urgent tasks, defining by the possibility of standardization of these power electronics solutions of high voltage, are:

1. Development of the optimal control algorithms for these devices on setting, adjusting and testing of their control systems;

2. Evaluation of the influence of these devices on steady-state and transient operating conditions of power systems, as well as on functioning of the relay protection and emergency automatic.

Due to the impossibility of making field experiments and tests of these devices in real power systems, the main tools for solving these problems are modeling and simulation. The listed tasks form the following specific requirements for simulation systems necessary for their solution:

Numerical investigations of heat and mass transfer processes at the ignition of solid [1-4], liquid [5-8] condensed substances and polymer materials [9, 10] by local energy source with limited power consumption (metallic and non-metallic particles, wires, cores, concentrate radiation flows and etc.) was held in recent years.

\footnotetext{
${ }^{\text {a }}$ Corresponding author: hecn@tpu.ru
} 
1. The simulation should be implemented without process decomposition. This is vital for adequate analysis of the full range of processes taking place in the power system and mutual influence of all power elements and phenomena;

2. The simulation should be conducted in real time and include the opportunity to interact with external devices (for testing and adjusting of control devices).

Existing digital real time simulation systems remarkably fit the second requirement and their application for hardware in the loop testing of protection and control units is quite effective. But a simulation of the full range of power system processes without their decomposition and during unlimited time period is out of capabilities of digital simulators. This fact is confirmed by the experience of large power companies and manufacturers $[1,2]$.

To solve the specified problem, we propose to choose a concept of real time hybrid simulation developed in Tomsk Polytechnic University meeting both of the above requirements. This concept allows developing multipurpose models of different power system elements, which can be automatically configured according to the parameters of real equipment. To develop such models of power electronic based devices algorithms and structures of converters, based on different power semiconductor switches [3,4] have been defined. The requirements and approaches to performing those devices in hybrid real time models have been formulated. And finally appropriate experiments have been conducted to prove the versatility of the developed models.

Only fragment of the results of this research and modeling in the software environment MATlab Simulink are presented in the format of this work.

\section{Principle of hybrid real time simulation approach}

The basic provisions of the approach:

1. Power equipment of EPS is described by mathematical models implemented via hybrid coprocessors (HCP).

2. Methodically accurate with guaranteed instrumental error solution of differential equation systems carried out by means of the continuous implicit integration method.

3. All types of commutation of power equipment and ensuring unlimited expandability of simulated EPS carried out on a physical level model.

4. The interconnection between the physical and mathematical modeling levels, control parameters of the simulated equipment and different switching elements are provided by means of appropriate voltage-current converters.

5. Models of automatic control and relay protection systems are implemented via a microprocessor units (MPU), containing a central processing unit (CPU) and functionally oriented peripheral processors (PP), as well as Server depending on the required composition of the input signals and the speed of algorithms execution.

The given concept is realized as a specialized software and hardware hybrid complex - Hybrid Real-Time Simulator (HRTSim) of EPS [5, 6].

A specialized hybrid processor (SHP) is the basic element of the modular structure of the HRTSim and provides an adequate comprehensive simulation in the real-time of power equipment models, as well as control and protection systems.

The basic structure of the SHP of the VSC model is shown in Fig. 1. 


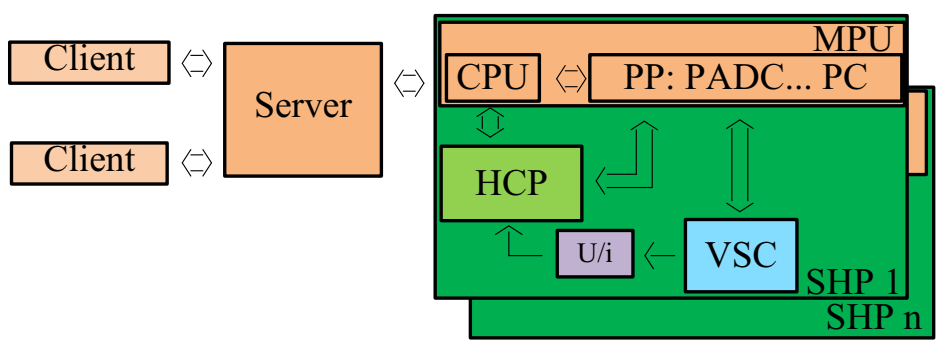

Figure 1. The structural diagram of the hybrid software and hardware simulation of EPS: SHP - specialized hybrid processor, CPU - central processing unit; PADC - processor of analog-to-digital converter; HCP - hybrid coprocessor; PC - switching processor; U/i - voltage-current converter; VSC - physical model of the power semiconductors VSC topology.

\section{The VSC simulation results}

According of the shown hybrid approach the models of power switches are implemented on a physical level via integrated microelectronic digital controlled analog switches (DCAS). At the same time to provide similarity of the model to a real device and to simulate any type of power electronic switches, including anti-parallel pair of different switches the commutation algorithms accounting input and output current and voltage values, as well as gate control signal are developed and implemented in MPU of SHP.

The switching time (t) of DCAS is about $300 \mathrm{~ns}$ [7], while a switching time of IGBT is more then $2 \mu s$, as a result the DCAS can be considered an Ideal Switch.

Consequently, the equivalent circuit of DCAS can be adapted to simulate the real power switches. Analysis of equivalent circuits of DCAS and real IGBT (type № 5SNR), a comparison of their parameters, taking into account modal and technical scaling coefficients were carried out to verify the adequacy of this simulation. The character of the transition process can be adapted by appropriate selection of parameters and variation of the equivalent circuit depending on type of simulated power semiconductors.

In Fig. 2 the scheme and commutation process simulation of IGBT (type № 5SNR) with the snubber circuit presented in [8] are shown.

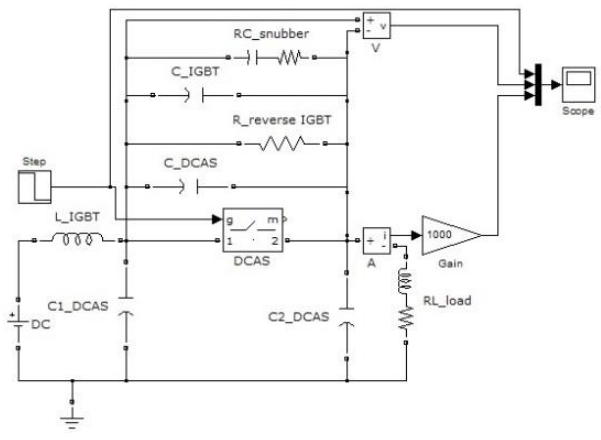

a)

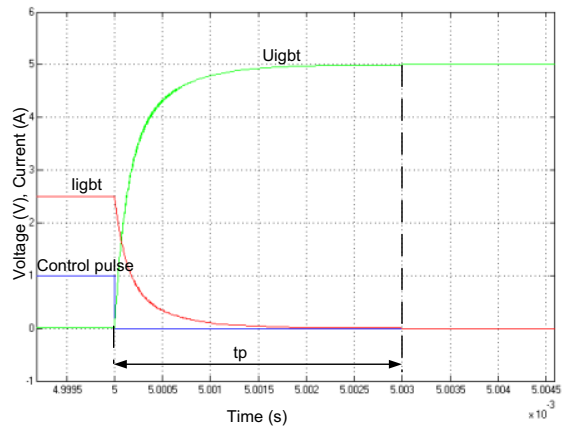

b)

Figure 2. The structural diagram of the hybrid software and hardware simulation of EPS: SHP - specialized hybrid processor, CPU - central processing unit; PADC - processor of analog-to-digital converter; HCP - hybrid coprocessor; PC - switching processor; U/i - voltage-current converter; VSC - physical model of the power semiconductors VSC topology.

In Fig. 2 the switching time (tp) is about $3 \mu \mathrm{s}$, corresponding the switching time of real IGBT (the recovering time of real 5SNR IGBT is about $1.7 \mu s$ [8]). The difference may be caused by errors in 


\section{MATEC Web of Conferences}

the recalculation of the parameters of the 5SNR IGBT or parameters the DC circuit that successfully solved by selection of elemental base and components.

The obtained IGBT scheme was tested on a standard bridge inverter (Fig.3). There is equivalent circuit of DCAS and IGBT is assembled in a box IGBT 1 and IGBT 2.

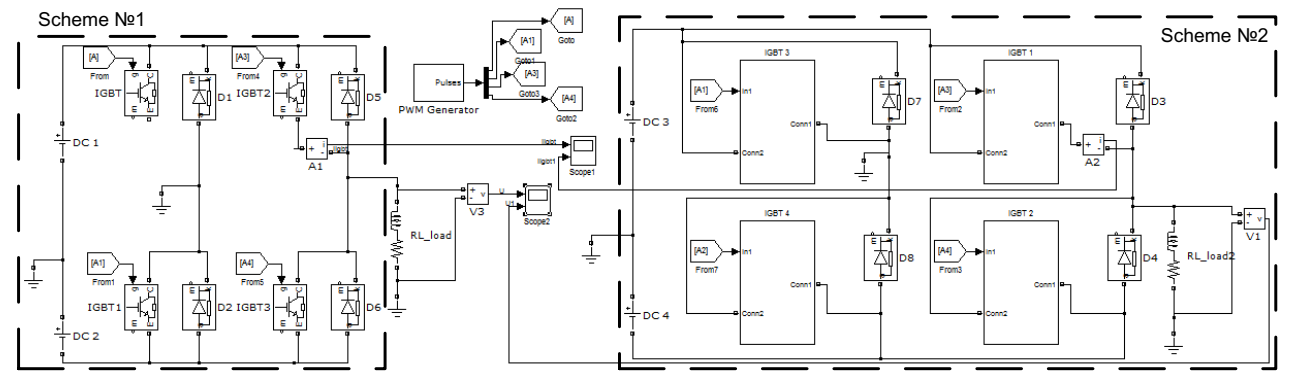

Figure 3. The scheme of standard bridge inverter: Scheme №1 includes a standard MATlab`s unit of the transistor, Scheme №2 includes an equivalent circuits of DCAS and IGBT.

In Fig. 4 current (a) and voltage (b) waveforms the scheme of bridge inverter are shown.

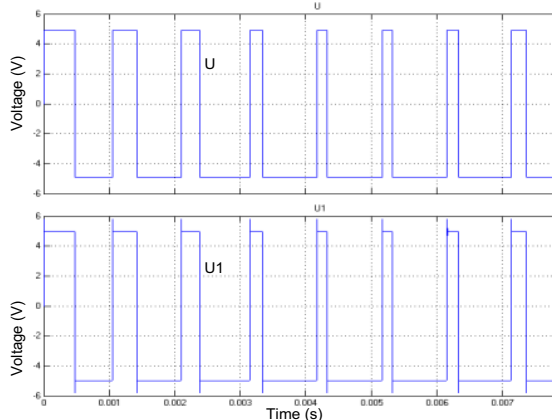

a)

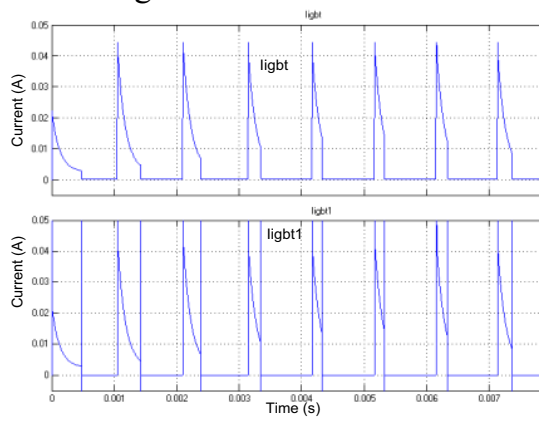

b)

Figure 4. The current (a) and voltage (b) waveforms of the scheme of bridge inverter.

In Fig. 5 spectral analysis of voltage waveforms the scheme of bridge inverter are shown.

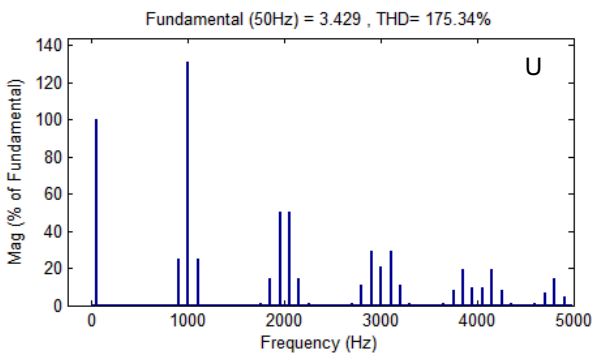

a)

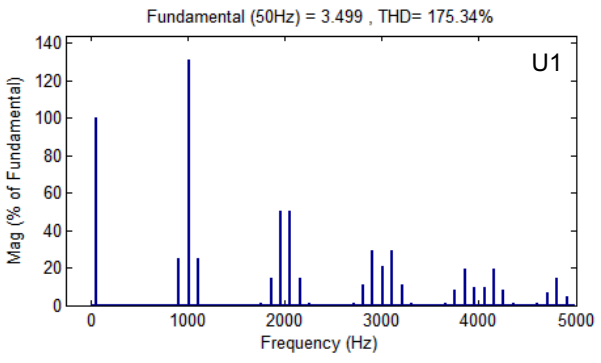

b)

Figure 5. The spectral analysis of voltage waveforms for the Scheme №1 (a) and the Scheme №2 (b).

The presented results confirm the adequacy of computer testing and adaptability developed equivalent circuits of physical modeling power semiconductors via DCAS and the possibility of their application for the accurate simulation of different switching processes, including VSC HVDC and FACTS devices, implemented within the framework of the specialized concept and tools of the hybrid simulation of real EPS. 


\section{Conclusions}

The proposed specialized hybrid concept and tools of the power semiconductors, as well as VSC of HVDC and FACTS devices, and EPS on a whole allow to carry a comprehensive real-time simulation on an unlimited range of a single continuous range of various quasi-steady-state and transient processes in equipment and real three-phase power system.

This work was supported by State Task «Science» 2.1318.2014 «Research and Development of Hybrid Simulator of Power System with Active Adaptive Networks».

\section{References}

1. V.K. Agrawal, R.K. Porwal, R. Kumar, P. Vivek, T. Muthukumar. In.Con. ICPS 6, 1-6 (2011)

2. Qi Dacai. ELECTRA 257, 4-16 ( 2011)

3. K. S. Vijay, HVDC and FACTS Controllers: Applications of Static Converters in Power Systems (Kluwcr Academic Publishers, USA, 2004)

4. B. R. Andersen VSC Transmission Tutorial held at the 2005 (CIGRE B4, (2005)

5. A. Prokhorov, Yu. Borovikov, A. Gusev, IEEE-ISGT 5, 1-5 (2013)

6. A. Gusev, Yu. Khrushchov, S. Svechkarev, I. Plodisty, IFOST 5, 1-5 (2009)

7. Maxim Integrated [Online] http://datasheets.maximintegrated.com/en/ds/MAX4661MAX4663.pdf

8. L. Max, Energy Evaluation for DC/DC Converters in DC-Based Wind Farms (Goteborg, Sweden 2007) 